OPEN ACCESS

Edited by:

Antonella Conte,

Sapienza University of Rome, Italy

Reviewed by:

Gina Ferrazzano,

Sapienza University of Rome, Italy

Christian Dresel,

Johannes Gutenberg University

Mainz, Germany

*Correspondence:

Yi-Jen Guo

ifiaa@yahoo.com.tw

Specialty section:

This article was submitted to

Movement Disorders,

a section of the journa

Frontiers in Neurology

Received: 17 August 2021 Accepted: 23 November 2021 Published: 15 December 2021

Citation:

Fang T-C, Chen C-M, Chang M-H, Wu C-H and Guo Y-J (2021) Altered

Functional Connectivity and Sensory

Processing in Blepharospasm and Hemifacial Spasm: Coexistence and Difference. Front. Neurol. 12:759869.

doi: 10.3389/fneur.2021.759869

\section{Altered Functional Connectivity and Sensory Processing in Blepharospasm and Hemifacial Spasm: Coexistence and Difference}

\author{
Ting-Chun Fang ${ }^{1}$, Chun-Ming Chen ${ }^{2}$, Ming-Hong Chang ${ }^{1,3}$, Chen-Hao Wu ${ }^{4}$ and \\ Yi-Jen Guo ${ }^{1 *}$
}

${ }^{1}$ Department of Neurology, Taichung Veterans General Hospital, Taichung City, Taiwan, ${ }^{2}$ Department of Medical Imaging, China Medical University Hospital, Taichung City, Taiwan, ${ }^{3}$ College of Life Science, National Chung Hsing University, Taichung City, Taiwan, ${ }^{4}$ Department of Radiology, Taichung Veterans General Hospital, Taichung City, Taiwan

Background: Blepharospasm (BSP) and hemifacial spasm (HFS) are both facial hyperkinesia however BSP is thought to be caused by maladaptation in multiple brain regions in contrast to the peripherally induced cause in HFS. Plausible coexisting pathophysiologies between these two distinct diseases have been proposed.

Objectives: In this study, we compared brain resting state functional connectivity (rsFC) and quantitative thermal test (QTT) results between patients with BSP, HFS and heathy controls (HCs).

Methods: This study enrolled 12 patients with BSP, 11 patients with HFS, and $15 \mathrm{HCs}$. All subjects received serial neuropsychiatric evaluations, questionnaires determining disease severity and functional impairment, QTT, and resting state functional MRI. Image data were acquired using seed-based analyses using the CONN toolbox.

Results: A higher cold detection threshold was found in the BSP and HFS patients compared to the HCs. The BSP and HFS patients had higher rsFC between the anterior cerebellum network and left occipital regions compared to the HCs. In all subjects, impaired cold detection threshold in the QTT of lower extremities had a correlation with higher rsFC between the anterior cerebellar network and left lingual gyrus. Compared to the HCs, increased rsFC in right postcentral gyrus in the BSP patients and decreased rsFC in the right amygdala and frontal orbital cortex in the HFS subjects were revealed when the anterior cerebellar network was used as seed.

Conclusions: Dysfunction of sensory processing detected by the QTT is found in the BSP and HSP patients. Altered functional connectivity between the anterior cerebellar network and left occipital region, especially the Brodmann area 19, may indicate the possibility of shared pathophysiology among BSP, HFS, and impaired cold detection threshold. Further large-scale longitudinal study is needed for testing this theory in the future.

Keywords: blepharospasm, hemifacial spasm, functional MRI, quantitative thermal test, resting state functional connectivity 


\section{INTRODUCTION}

Blepharospasm (BSP) is a focal dystonia characterized by bilateral, synchronous contractions of the orbicularis oculi. The etiology of BSP is unknown in most cases, but it may coexist with Parkinson's disease, progressive supranuclear palsy and multiple system atrophy, or it may be associated with lesions of the brainstem, thalamus, and basal ganglion (1). Neurophysiological and neuroimaging evidence suggests that BSP involves multiple brain regions including the basal ganglion, cerebellum, cerebral cortex, thalamus, and brainstem (2-5). Dysfunction within the sensorimotor system in BSP has been found. Overactivity of the postcentral gyrus and supplementary motor area were reported during whistling in BSP patients (6). One study showed reduced resting-state functional connectivity (rsFC) between sensorimotor cortex and several seeds including the caudate, cingulate gyrus, and cerebellum; besides, rsFC changes between the cerebellum and occipital cortex were also found, which suggest their possible functional role in sensory information or visuomotor integration in BSP (7). Defects in sensorimotor integration, loss of inhibition and maladaptive plasticity, may lead to BSP (2). Hemifacial spasm (HFS) is also a facial dyskinesia with unilateral paroxysmal contractions of facial muscles innervated by ipsilateral facial nerve. The most common etiology of HFS is compression at the root exit zone of facial nerve by aberrant vessels leading to spontaneous ephaptic nerve depolarizations in the root entry zone (8). HFS is considered to be a peripherally induced disorder; however, the antidromic transmission of stimuli may cause central and cortical reorganization $(9,10)$. In addition, atrophic amygdala and altered $\mathrm{rsFC}$ from the amygdala to the medial prefrontal cortex, orbital frontal cortex, and posterior insula have been reported, suggesting that the central mechanism of HFS may be associated with the disease severity and anxiety (11). Although BSP and HFS seem to be distinct diseases, a possible overlapping pathophysiologic mechanism is proposed (12). A study of structural brain networks also revealed increased global network segregation, decreased global efficiency and reduced degree of local structural network in the cerebella of both BSP and HFS patients (13).

Alterations of proprioceptive, tactile, nociceptive, and thermal information processing may be related to altered sensorimotor integration in focal dystonia, including blepharospasm (14). The somatosensory abnormalities are not only present in the body part affected by dystonia, but may also extend to the unaffected body parts (15-17). Quantitative thermal test (QTT) is a method to detect cold and heat detection thresholds. Previous study using quantitative sensory test reported impaired thermal detection threshold in patients with idiopathic focal dystonia (17).

Most previous functional studies have discussed BSP and HFS separately. In this study, we aimed to elucidate the correlations and differences of rsFC between BSP and HFS, by using known networks as seeds from the functional MRI (fMRI). We proposed that there might be altered connections between the cerebellum, sensorimotor cortex, and occipital lobe as the results reported by previous studies $(6,7,13)$. We also hypothesized that there may be differences in sensory processing between patients with
BSP, HFS, and healthy controls (HCs), which could be detected by the QTT.

\section{MATERIALS AND METHODS}

\section{Subjects}

A total of 38 subjects were studied, including 12 patients with BSP (disease duration of $8.33 \pm 5.30$ years), 11 patients with HFS (disease duration of $8.50 \pm 3.68$ years), and 15 HCs. The patients with BSP and HFS were under regular treatment of local injection of botulinum toxin (BTX), with mean treatment duration of $3.83 \pm 0.94$ years in the BSP group, and $3.83 \pm$ 1.27 years in the HFS group. The study was approved by the Taichung Veteran General Hospital Research Ethical Board in Taichung, Taiwan (CE16282B), and all participants provided written informed consent before participating in the study. All methods were performed in accordance with the Declaration of Helsinki guidelines and hospital regulations.

\section{Clinical Assessment}

The QTT was performed in all subjects to measure warm and cold thresholds in 4 limbs using the PATHYWAY systems (Medoc, Ramat Yishay, Israel). A thermode of the device which could heat up or cool down from the baseline of $32^{\circ} \mathrm{C}$ was placed on the subject's skin. The subject then gave a response to the examiner when they felt the slightest temperature change (either warmer or colder), and the temperature was recorded. This was repeated three times each for warm and cold, and the mean temperatures were calculated as the warm and cold detection thresholds.

The patients with BSP were evaluated with the Jankovic Rating Scale (JRS) and the Blepharospasm disability index (BSDI). The JRS contains two subscales of severity and frequency, both of which are scored on a 5-point scales ranging from 0 to 4 , with 4 indicating the most severe or frequent symptom. The BSDI represents the impairment of six daily activities related to BSP. Each activity is rated from 0 to 4 , with 4 indicating that it is not possible to perform the activity due to disease (18).

The patients with HFS were evaluated using a 5-point rating scale for symptom severity and seven self-rating items (HFS7) for health-related quality of life. The 5-point rating scale was scored from 0 to 4 , with a higher score indicating more severe symptoms. The HFS-7 questionnaire contains seven items regarding activities of daily living, emotional well-being, and stigma. All items are self-rated from 0 (never) to 4 (always) (19).

In addition, all of the participants received the Mini-Mental State Examination (MMSE) to evaluate cognition, and the Beck Depression Inventory II (BDI-II) and Beck Anxiety Inventory (BAI) to assess mood status.

\section{MRI Data Acquisition}

For the MRI examination, the patients wore earplugs and headsets before entering the scan room. Inappropriate items such as ferromagnetic materials and electronic devices were removed for safety. During the resting-state scan, the participant was instructed to remain awake with their eyes closed, lie still, and think of nothing. Illumination was kept low to improve patient 
comfort. The first four volumes were considered as dummy scans and discarded automatically by the scanner.

Data were collected on a Philips 3T scanner (Achieva, Philips Healthcare, Best, Netherlands). A gradient echo-planar sequence with axial orientation was used to collect functional data (TR $=2000 \mathrm{~ms}, \mathrm{TE}=30 \mathrm{~ms}$, flip angle $=90^{\circ}, 40$ slices with $3 \mathrm{~mm}$ thickness and $1 \mathrm{~mm}$ gap, voxel size $=3 \times 3 \times 3 \mathrm{~mm}^{3}$. A highresolution T1-weighted MPRAGE $(\mathrm{TR}=8 \mathrm{~ms}$, TE $=3.7 \mathrm{~ms}$, flip angle $=15^{\circ}, 168$ slices, voxel size $=1.0 \times 1.0 \times 1.0 \mathrm{~mm}^{3}$ ) was obtained.

\section{MRI Data Analysis}

Preprocessing of fMRI data was performed using Functional Connectivity Toolbox (CONN) pipeline version 18.b (www.nitrc.org/projects/conn, RRID:SCR_009550). First, slice-timing correction was performed. The slices were acquired in ascending, interleaved order, and the images were realigned to correct for subject head motion. Functional and structural segmentations were done to divide the data into different tissue classes: white matter, gray matter, and cerebrospinal fluid (CSF). The images were then normalized to a Montreal Neurological Institute template, and the normalized images were resliced with a target resolution of $2 \mathrm{~mm}$. The normalized fMRI images were then smoothed with an 8 -mm full width at half maximum Gaussian kernel. The motion outlier threshold of artifact detection tool was set at 95th percentiles in normative sample with motion threshold $0.5 \mathrm{~mm}$. In the preprocessing pipeline,
ART-based identification was applied, and acquisitions with displacement above the threshold were removed. Brain masking process was applied for voxel-level analysis. A band-pass filter from $0.01 \mathrm{~Hz}$ to infinity were used.

To investigate functional connectivity, we performed seed-tovoxel analysis to our acquiring data. For the seeds selection, we chose all pre-defined seeds of the CONN toolbox that belong to functional networks, including (1) Default Mode Network (4 ROIs), (2) Frontoparietal Network (4 ROIs), (3) Sensorimotor Network (3 ROIs), (4) Salience Network (7 ROIs), (5) Dorsal Attention Network (4 ROIs), (6) Visual Network (4 ROIs), (7) Language Network (4 ROIs), and (8) Cerebellar Network (2 ROIs) (Supplementary Figure S1). All results were displayed under the significance of $p=0.001$ (uncorrected, peak-level) and $p=0.05$ (false discovery rate (FDR) corrected, clusterlevel). To compare the effects between the BSP patients and HCs and between the HFS patients and HCs, analysis of covariance (ANCOVA) was used to control for covariates (age and gender) in the CONN toolbox. Multiple regression analysis was used to analyze the unique effect of temperature threshold, which was also adjusted for age and gender.

\section{Other Statistical Analysis}

Clinical characteristics between the groups were compared using the Kruskal-Wallis test as a non-parametric test for continuous variables, and the chi-square test for binary variables. All statistical analyses were performed using SPSS version 20

TABLE 1 | Clinical characteristics of the study subjects.

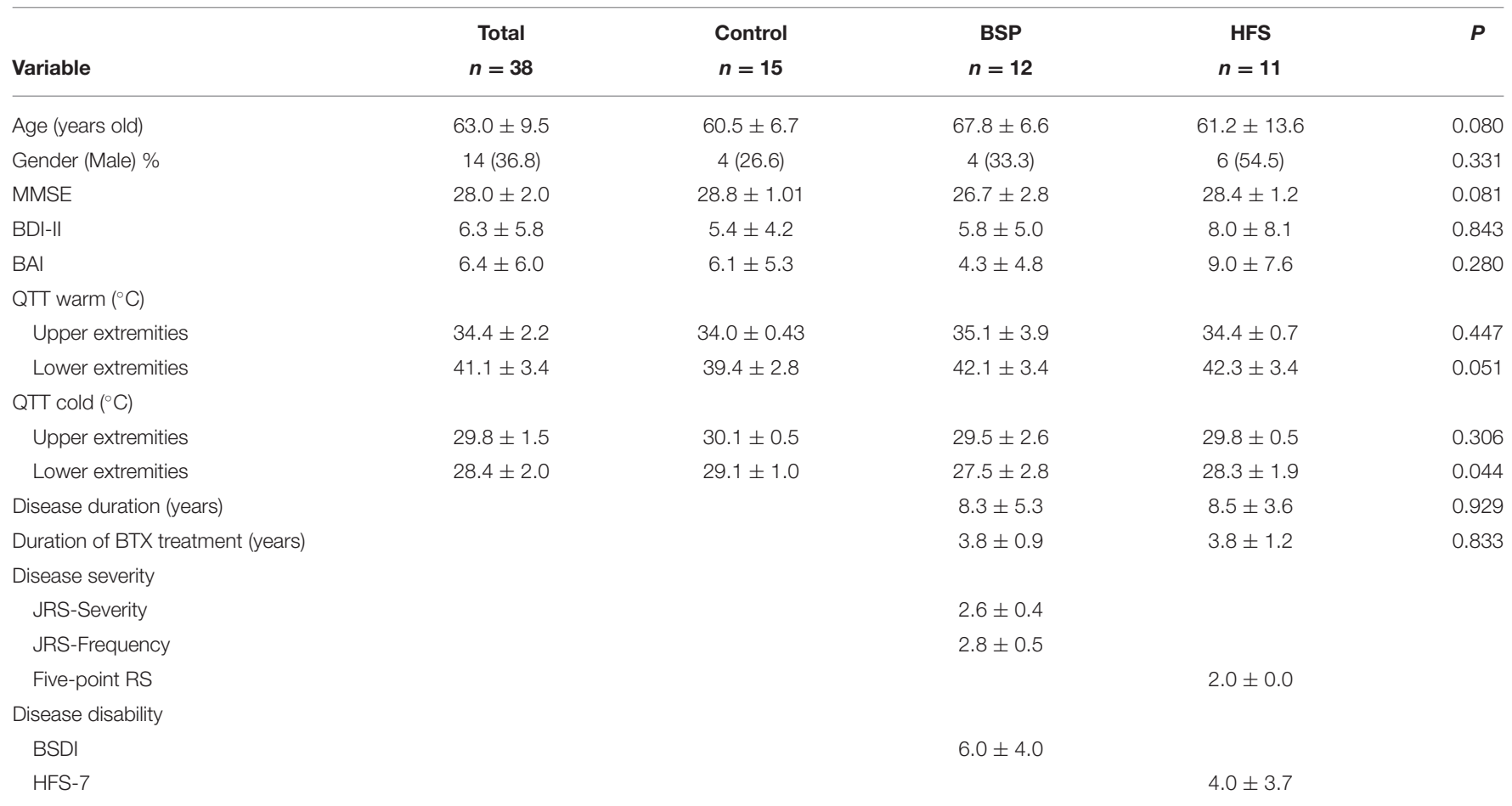

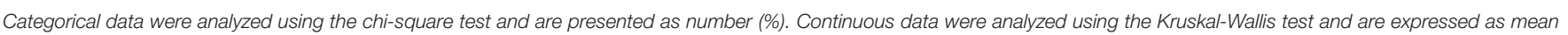

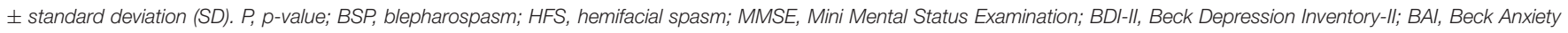
Inventory; QTT, quantitative thermal test; BTX, botulinum toxin; JRS, Jankovic Rating Scale; Five-point RS, 5-point rating scale; BSDI, Blepharospasm Disability Index. 
TABLE 2 | Increased functional connectivity in different groups for seed ROI with the anterior cerebellar network.

\begin{tabular}{|c|c|c|c|c|c|}
\hline Groups & Target & Functional area & $\begin{array}{l}\text { Peak MNI coordinate } \\
\text { (x y z) }\end{array}$ & $\begin{array}{l}\text { Cluster size } \\
\text { (voxel) }\end{array}$ & $\boldsymbol{P}$ \\
\hline \multirow[t]{3}{*}{ BSP vs. HC } & $\begin{array}{l}\text { Postcentral and precentral } \\
\text { gyrus R }\end{array}$ & $\begin{array}{l}\text { Primary somatosensory and motor cortex } \\
\text { (BA 1-3, 4) }\end{array}$ & $+56-10+32$ & 139 & 0.0000 \\
\hline & Postcentral gyrus R & Primary somatosensory cortex (BA 1-3) & $+16-34+64$ & 119 & 0.0001 \\
\hline & Cuneal cortex L & Associative visual cortex (BA 19) & $-8-80+30$ & 119 & 0.0000 \\
\hline HFS vs. HC & $\begin{array}{l}\text { Inferior and superior lateral } \\
\text { occipital cortex } L\end{array}$ & $\begin{array}{l}\text { Secondary and associative visual cortex } \\
\text { (BA 18, 19) }\end{array}$ & $-40-78+04$ & 165 & 0.0000 \\
\hline $\begin{array}{l}\text { Effect of QTT scores (lower } \\
\text { extremities) }\end{array}$ & $\begin{array}{l}\text { Lingual gyrus, Occipital } \\
\text { fusiform gyrus, Cerebellum } \\
4 \text { and } 5 \text {, Cerebellum } 6 \text { L }\end{array}$ & $\begin{array}{l}\text { Secondary and associative visual cortex } \\
\text { (BA 18, 19) }\end{array}$ & $-18-62-10$ & 387 & 0.0000 \\
\hline
\end{tabular}

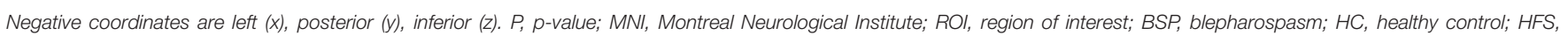
hemifacial spasm; QTT, quantitative thermal test; R, Right; L, Left; BA, Brodmann area.

(IBM Corporation, Armonk, New York, USA), and statistical significance levels were set at $p<0.05$.

\section{RESULTS}

\section{Demographic Information}

There was a significant difference in cold detection threshold of the QTT over the lower extremities between the three groups ( $p$ $=0.044$ ) (Table 1). Post hoc analysis revealed that the BSP and HFS groups had a higher cold detection threshold than the HC group, but there was no significant difference between the BSP and HFS groups. There were no significant differences in other QTT test parameters including warm detection threshold over the upper and lower extremities and cold detection threshold over the upper extremities. In addition, there were no significant differences in age, gender, disease duration, duration of BTX treatment, MMSE, BDI-II, or BAI between the three groups.

\section{Resting-State fMRI, Seed-to-Voxel Functional Connectivity}

Table 2 reveals that the BSP group demonstrated an increased functional connectivity between the anterior cerebellar network and left cuneal cortex (Broadmann area 19, BA19) when comparing to the HC group after adjusting for age and gender (Figure 1A, left cuneal cortex, $p$ uncorrected $=0.000026, p$ $F D R=0.000072)$. Increased functional connectivity between the anterior cerebellar network and left lateral occipital cortex (BA19) was found in the HFS group when comparing to the HC group after adjusting for age and gender (Figure 1B, lateral occipital cortex, $p$ uncorrected $=0.000024, p$ FDR $=0.000024$ ). Increased connectivity between the anterior cerebellar network and left lingual gyrus (BA19) was correlated with an impaired cold detection threshold over the lower extremities after adjusting for age and gender in all subjects (Figure 1C, $p$ uncorrected $=$ $0.000004, p F D R=0.000004)$.

Table 2 reveals that the BSP group demonstrated an increased connectivity between the anterior cerebellar network and right precentral and postcentral gyri after adjusting for age and gender relative to the $\mathrm{HC}$ group (Figure 2, $p$ uncorrected $=$
0.000048, $p F D R=0.000072$, and $p$ uncorrected $=0.00012$, $p F D R=0.00012$, respectively).

Table 3 reveals that there was reduced connectivity between the anterior cerebellar network and right frontal orbital cortex after adjusting for age and gender in the HFS group when comparing to the HC group (Figure 3, p uncorrected $<0.000001$, p FDR < 0.000001).

\section{DISCUSSION}

This study suggests that the cerebellum plays an role in the central network disturbance in BSP and HFS. Increased rsFC between the anterior cerebellar network and left occipital regions (BA19) was found in both BSP and HFS groups (Figures 1A,B,D). Increasing evidence suggests that network deficits in the cerebellum, thalamus, and cortex result in altered sensorimotor integration, leading to $\operatorname{BSP}(2,20,21)$. Although HFS is mostly caused by peripheral irritation, a previous study using structural MRI reported reduced local connectivity in the cerebellum in both HFS and BSP patients (13). Moreover, our data showed that there was increased rsFC between the anterior cerebellar network and left lingual lobe (BA19), which correlated with impaired cold detection threshold over lower extremities in all subjects (Figures 1C,D). This finding may support the perspective that the cerebellum acts as a center of clusters processing proprioception, discrimination, and somatosensory thresholds, and it also underlies non-nociceptive processes such as attention and decision-making which predicts the pain (20, 22).

In this study, cold threshold test showed that the BSP and HFS patients were more insensitive to coldness in lower limbs than the HC group. This suggested that altered somatosensory processing may play a role in both BSP and HSP. The pathophysiology of dystonia involves defects in sensory or perceptual processing (2). Most previous studies using functional imaging have reported altered activity in the sensorimotor cortex (SMC) in patients with dystonia $(23,24)$. One study of patients with BSP reported increased activity over the SMC when whistling (6). Another 


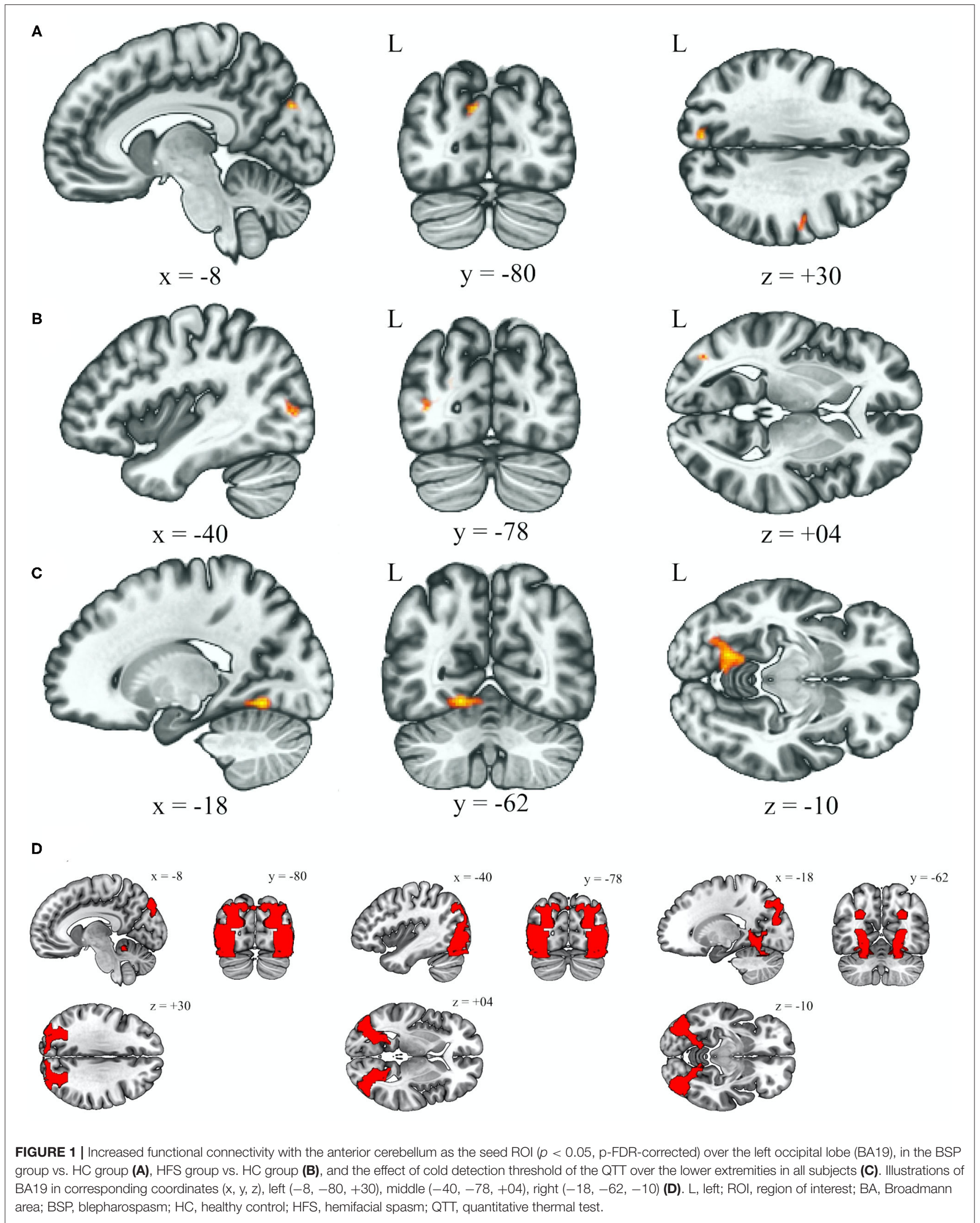


A
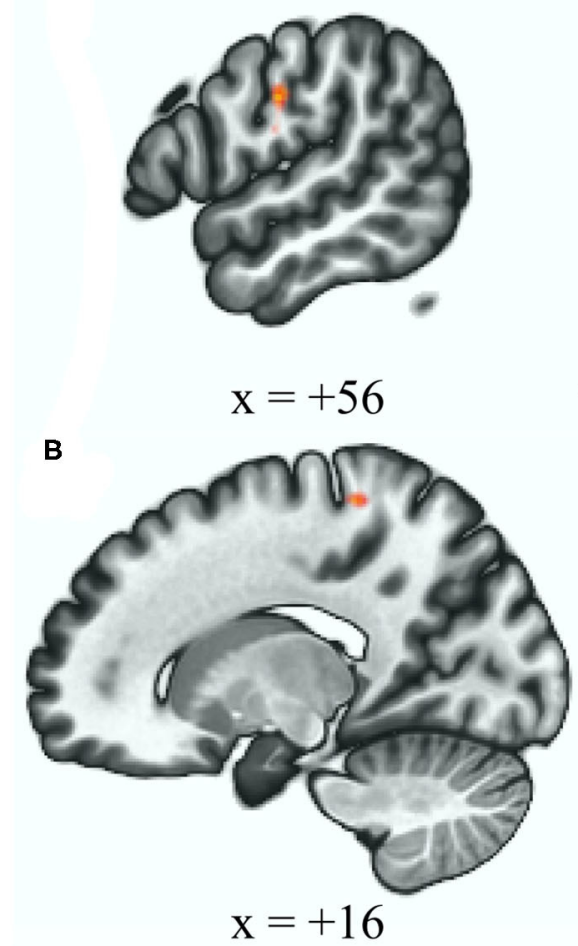

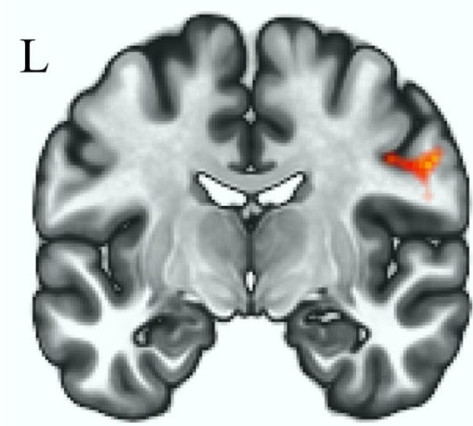

$\mathrm{y}=-10$

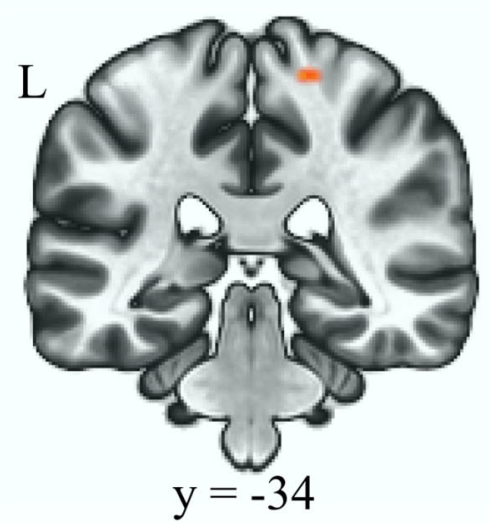

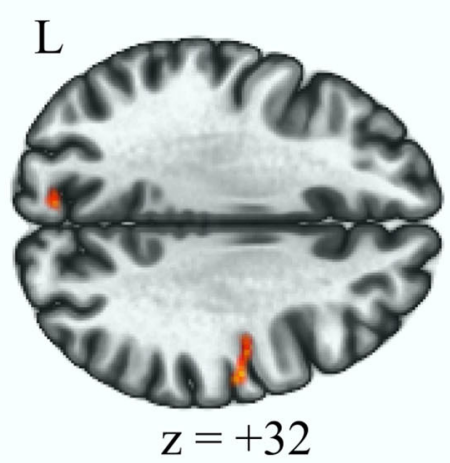

L

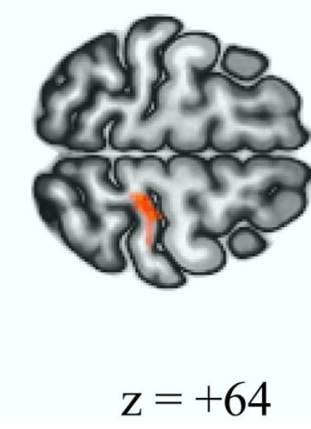

FIGURE 2 | Increased functional connectivity with the anterior cerebellum as the seed ROI $(p<0.05, p$-FDR-corrected) in the BSP group vs. HC group, over the right postcentral and precentral gyrus (A), and over the right postcentral gyrus (B). L, left; ROI, region of interest; BSP, blepharospasm; HC, healthy control.

TABLE 3 | Decreased functional connectivity in different groups for seed ROI with the anterior cerebellar network.

\begin{tabular}{|c|c|c|c|c|c|}
\hline Groups & Target & Functional area & $\begin{array}{l}\text { Peak MNI coordinate } \\
\text { ( } x \text { y } z)\end{array}$ & Cluster size (voxel) & $P$ \\
\hline HFS vs. HC & $\begin{array}{l}\text { Frontal orbital cortex, amygdala, } \\
\text { Parahippocampal gyrus R }\end{array}$ & $\begin{array}{l}\text { Orbitofrontal and subgenual area (BA 11, } \\
25) \text {, dorsal entorhinal cortex (BA 34) }\end{array}$ & $+14+02-22$ & 340 & 0.0000 \\
\hline
\end{tabular}

Negative coordinates are left (x), posterior (y), inferior (z). P, p value; MNI, Montreal Neurological Institute; ROI, region of interest; HC, healthy control; HFS, hemifacial spasm; R, Right; $B A$, Brodmann area.

resting fMRI study also reported decreased rsFC in the SMC to several seeds (7). Our data revealed that impaired cold threshold detection in lower extremities was associated with increased rsFC between left lingual lobe and anterior cerebellar network, and therefore supports the view that lingual gyrus may be involved in somatosensory stimulation and perception of sensory stimuli (25). In addition, the occipital lobe has been reported to be a pain processing brain network in previous studies $(22,26-28)$. One study investigating pain sensitivity with pain-free resting-state functional brain connectivity also suggested that several connections involving the occipital lobe influence the prediction of pain sensitivity (29). These findings suggest that dealing with the sensory information is possibly one of the functions of the occipital lobe. When comparing to the control group, both the BSP and HFS groups has increased rsFC between the left occipital regions (BA19) and anterior cerebellar network. We suppose that BA19, being part of occipital lobe and composed of the lingual gyrus, cuneus, and lateral occipital gyrus, may have its functional role in BSP, HFS and sensory processing. Considering with the interconnection within the occipital lobe $(25,30)$, altered sensory integration in the occipital lobe might be one of the plausible explanations for the rsFC within BA19 and impaired thermal detection in both BSP and HFS. Clinically BSP can result in functional blindness. The converted occipital function is probably related to the interruption of visual inputs, as glucose hypometabolism in the visual cortex has been observed in BSP $(7,31)$. Alternatively, activation of the visual cortex in BSP may suggest that the visuomotor pathway mediates facial hyperkinesia such as blinking to visual stimuli (32). Nevertheless, altered 

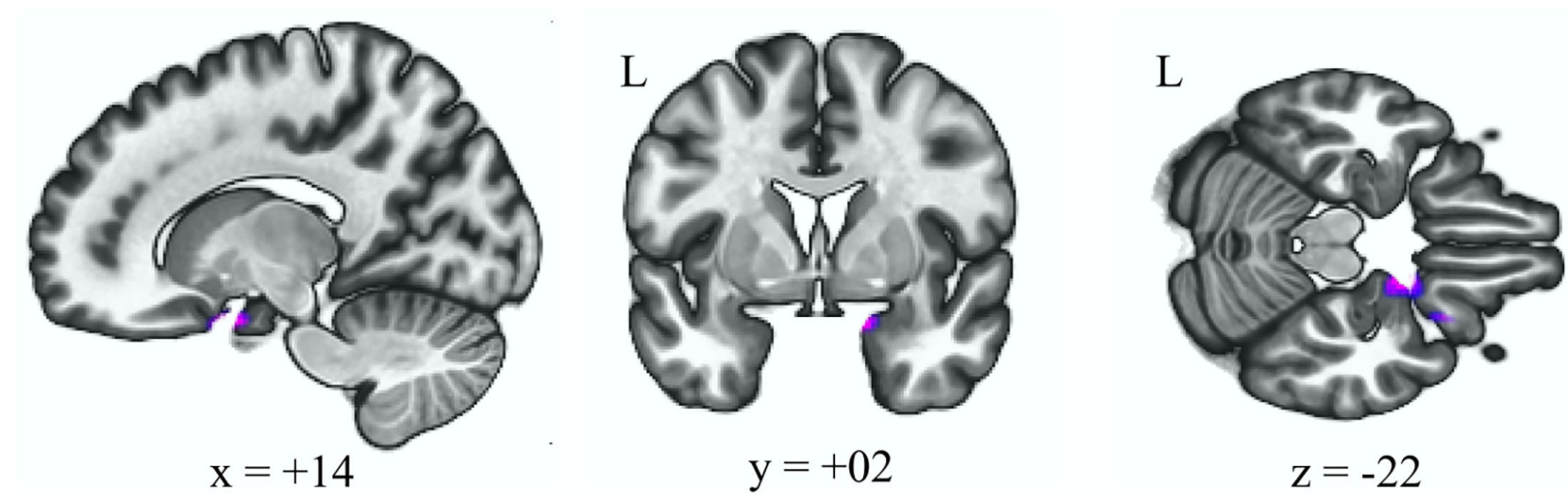

FIGURE 3 | Decreased functional connectivity with the anterior cerebellum as the seed ROI ( $p<0.05$, p-FDR-corrected) in the HFS group vs. HC group. L, left; ROI, region of interest; HFS, hemifacial spasm; HC, healthy control.

functional connectivity of the cerebellar visual network has also been shown in patients with other focal task-specific dystonia such as writer's cramp or embouchure dystonia which do not involving blinking (33).

In this study, some differences in the altered rsFC were revealed between the BSP and HFS groups. With the cerebellum as the seed ROI, the BSP group showed increased rsFC in the somatosensory and motor cortex, while the HSP showed decreased rsFC in the orbitofrontal cortex and amygdala. Changes in the activation level in the sensorimotor cortex have generally been reported in dystonia, including BSP $(2,20)$. Previous studies on resting state fMRI have shown decreased rsFC in the sensorimotor cortex within the sensorimotor network (34). Compared to the dysfunction of integration in the sensorimotor network, increased rsFC between the cerebellar network and sensorimotor cortex may explain the cerebellar modulation of sensorimotor plasticity (35). An atrophic amygdala together with increased rsFC of the amygdala to orbitofrontal cortex, medial prefrontal cortex, and insula have been reported in HFS patient (11). These abnormalities may lead to the emotional and visual deficiency, since the amygdala coordinates efferent and afferent information such as pain, anxiety, fear, reward, and visual information (36). In our study, although no difference in BAI was noted between the HSP and HFS groups, we speculate that the changes in rsFC between the cerebellar seed ROI, orbital frontal cortex, and amygdala may be a hint of the altered visuomotor or sensory processing in HFS.

There are several limitations to this study. First, although there were some statistically significant results in this study, our sample size was relatively small. Second, the information obtained from resting state functional images may not explain the causal relationship between clinical symptoms and alteration of brain functional communication Third, this study was a crosssectional design, and we were unable to observe alterations of functional state with disease progression over time. Further studies with more subjects, a longitudinal design, or using multimodalities should be performed.

In conclusion, the BSP and HFS patients have impaired cold detection threshold in lower extremities. Altered functional connectivity between the anterior cerebellar network and left occipital regions, especially the BA19, may be associated with BSP, HFS, and impaired cold detection threshold. The cerebellum and occipital lobe might have their functional role in BSP and HFS, and we postulate that impaired somatosensory processing may be one of the possible pathophysiological mechanisms. In addition, the differences in the rsFC to the anterior cerebellar network seed, including increased rsFC within the sensorimotor cortex in the BSP patients and decreased rsFC within the orbitofrontal cortex and amygdala in the HFS patients, may indicate clinical relevance to the disease entity. We believe that these findings provide a unique insight, and may help to understand and distinguish the pathophysiology between BSP and HFS in the future.

\section{DATA AVAILABILITY STATEMENT}

The raw data supporting the conclusions of this article will be made available by the authors, without undue reservation.

\section{ETHICS STATEMENT}

The studies involving human participants were reviewed and approved by Taichung Veteran General Hospital Research Ethical Board in Taichung, Taiwan. The patients/participants provided their written informed consent to participate in this study.

\section{AUTHOR CONTRIBUTIONS}

T-CF, C-MC, and Y-JG conceptualized the project. M-HC and $\mathrm{C}-\mathrm{HW}$ performed the data acquisition and investigation. T-CF wrote the first draft of the manuscript. Y-JG critically reviewed the manuscript. All authors contributed to writing and revising the manuscript. 


\section{FUNDING}

This work was supported by Taichung Veterans General Hospital [grant numbers 106DHA0500161].

\section{ACKNOWLEDGMENTS}

We thank the staff at the Neurological Institute in Taichung Veteran General Hospital for participant recruitment and clinical

\section{REFERENCES}

1. Grandas F, Elston J, Quinn N, Marsden CD. Blepharospasm: a review of 264 patients. J Neurol Neurosurg Psychiatry. (1988) 51:767-72. doi: 10.1136/jnnp.51.6.767

2. Neycher VK, Gross RE, Lehericy S, Hess EJ, Jinnah HA. The functional neuroanatomy of dystonia. Neurobiol Dis. (2011) 42:185-201. doi: 10.1016/j.nbd.2011.01.026

3. Fiorio M, Tinazzi M, Scontrini A, Stanzani C, Gambarin M, Fiaschi A, et al. Tactile temporal discrimination in patients with blepharospasm. $J$ Neurol Neurosurg Psychiatry. (2008) 79:796-8. doi: 10.1136/jnnp.2007.1 31524

4. Hutchinson M, Nakamura T, Moeller JR, Antonini A, Belakhlef A, Dhawan V, et al. The metabolic topography of essential blepharospasm: a focal dystonia with general implications. Neurology. (2000) 55:673-7. doi: 10.1212/WNL.55.5.673

5. Feiwell RJ, Black KJ, McGee-Minnich LA, Snyder AZ, MacLeod AM, Perlmutter JS. Diminished regional cerebral blood flow response to vibration in patients with blepharospasm. Neurology. (1999) 52:291-7. doi: 10.1212/WNL.52.2.291

6. Dresel C, Haslinger B, Castrop F, Wohlschlaeger AM, Ceballos-Baumann AO. Silent event-related fMRI reveals deficient motor and enhanced somatosensory activation in orofacial dystonia. Brain. (2006) 129(Pt 1):36-46. doi: 10.1093/brain/awh665

7. Jochim A, Li Y, Gora-Stahlberg G, Mantel T, Berndt M, Castrop F, et al. Altered functional connectivity in blepharospasm/orofacial dystonia. Brain Behav. (2018) 8:e00894. doi: 10.1002/brb3.894

8. Wang A, Jankovic J. Hemifacial spasm: Clinical findings and treatment. Muscle Nerve. (1998) 21:1740-7.

9. Rijntjes M, Tegenthoff M, Liepert J, Leonhardt G, Kotterba S, Muller S, et al. Cortical reorganization in patients with facial palsy. Ann Neurol. (1997) 41:621-30. doi: 10.1002/ana.410410511

10. Niu X, Xu H, Guo C, Yang T, Kress D, Gao L, et al. Strengthened thalamoparietal functional connectivity in patients with hemifacial spasm: a cross-sectional resting-state fMRI study. Br J Radiol. (2020) 93:20190887. doi: 10.1259/bjr.20190887

11. Xu H, Guo C, Li H, Gao L, Zhang M, Wang Y. Structural and functional amygdala abnormalities in hemifacial spasm. Front Neurol. (2019) 10:393. doi: $10.3389 /$ fneur.2019.00393

12. Tan EK, Chan LL, Koh KK. Coexistent blepharospasm and hemifacial spasm: overlapping pathophysiologic mechanism? J Neurol Neurosurg Psychiatry. (2004) 75:494-6. doi: 10.1136/jnnp.2003.0 19331

13. Chirumamilla VC, Dresel C, Koirala N, Gonzalez-Escamilla G, Deuschl $\mathrm{G}$, Zeuner KE, et al. Structural brain network fingerprints of focal dystonia. Ther Adv Neurol Disord. (2019) 12:1756286419880664. doi: $10.1177 / 1756286419880664$

14. Conte A, Defazio G, Hallett M, Fabbrini G, Berardelli A. The role of sensory information in the pathophysiology of focal dystonias. Nat Rev Neurol. (2019) 15:224-33. doi: 10.1038/s41582-019-0137-9

15. Quartarone A, Morgante F. Sant'angelo A, Rizzo V, Bagnato S, Terranova C, et al. Abnormal plasticity of sensorimotor circuits extends beyond the affected body part in focal dystonia. J Neurol Neurosurg Psychiatry. (2008) 79:985-90. doi: 10.1136/jnnp.2007.121632 studies, and the staff at the Department of Radiology in Taichung Veterans General Hospital for image acquisition.

\section{SUPPLEMENTARY MATERIAL}

The Supplementary Material for this article can be found online at: https:/www.frontiersin.org/articles/10.3389/fneur. 2021.759869/full\#supplementary-material

16. Defazio G, Berardelli A, Hallett M. Do primary adult-onset focal dystonias share aetiological factors? Brain. (2007) 130(Pt 5):1183-93. doi: 10.1093/brain/awl355

17. Paracka L, Wegner F, Blahak C, Abdallat M, Saryyeva A, Dressler D, et al. Sensory alterations in patients with isolated idiopathic dystonia: an exploratory quantitative sensory testing analysis. Front Neurol. (2017) 8:553. doi: 10.3389/fneur.2017.00553

18. Wabbels B, Jost WH, Roggenkamper P. Difficulties with differentiating botulinum toxin treatment effects in essential blepharospasm. J Neural Transm (Vienna). (2011) 118:925-43. doi: 10.1007/s00702-010-0546-9

19. Tan EK, Fook-Chong S, Lum SY, Thumboo J. Validation of a short disease specific quality of life scale for hemifacial spasm: correlation with SF-36. J Neurol Neurosurg Psychiatry. (2005) 76:1707-10. doi: 10.1136/jnnp.2005.065656

20. Lehericy S, Tijssen MA, Vidailhet M, Kaji R, Meunier S. The anatomical basis of dystonia: current view using neuroimaging. Mov Disord. (2013) 28:944-57. doi: $10.1002 / \mathrm{mds} .25527$

21. Yang J, Luo C, Song W, Chen Q, Chen K, Chen X, et al. Altered regional spontaneous neuronal activity in blepharospasm: a resting state fMRI study. $J$ Neurol. (2013) 260:2754-60. doi: 10.1007/s00415-013-7042-8

22. Atlas LY, Lindquist MA, Bolger $\mathrm{N}$, Wager TD. Brain mediators of the effects of noxious heat on pain. Pain. (2014) 155:1632-48. doi: 10.1016/j.pain.2014.05.015

23. Haslinger B, Altenmuller E, Castrop F, Zimmer C, Dresel C. Sensorimotor overactivity as a pathophysiologic trait of embouchure dystonia. Neurology. (2010) 74:1790-7. doi: 10.1212/WNL.0b013e3181e0f784

24. Wu CC, Fairhall SL, McNair NA, Hamm JP, Kirk IJ, Cunnington R, et al. Impaired sensorimotor integration in focal hand dystonia patients in the absence of symptoms. J Neurol Neurosurg Psychiatry. (2010) 81:659-65. doi: 10.1136/jnnp.2009.185637

25. Kong J, Kaptchuk TJ, Webb JM, Kong JT, Sasaki Y, Polich GR, et al. Functional neuroanatomical investigation of vision-related acupuncture point specificity-a multisession fMRI study. Hum Brain Mapp. (2009) 30:38-46. doi: $10.1002 / \mathrm{hbm} .20481$

26. Apkarian AV, Bushnell MC, Treede RD, Zubieta JK. Human brain mechanisms of pain perception and regulation in health and disease. Eur $J$ Pain. (2005) 9:463-84. doi: 10.1016/j.ejpain.2004.11.001

27. Boghi A, Rampado O, Bergui M, Avidano F, Manzone C, Coriasco M. et al. Functional MR study of a motor task and the tower of London task at $10 \mathrm{~T}$. Neuroradiology. (2006) 48:763-71. doi: 10.1007/s00234-006-0119-7

28. Baciu MV, Bonaz BL, Papillon E, Bost RA, Le Bas JF, Fournet J, et al. Central processing of rectal pain: a functional MR imaging study. AJNR Am J Neuroradiol. (1999) 20:1920-4.

29. Spisak T, Kincses B, Schlitt F, Zunhammer M, Schmidt-Wilcke T, Kincses ZT, et al. Pain-free resting-state functional brain connectivity predicts individual pain sensitivity. Nat Commun. (2020) 11:187. doi: 10.1038/s41467-019-13785-z

30. Palejwala AH, Dadario NB, Young IM, O'Connor K, Briggs RG, Conner AK, et al. Anatomy and white matter connections of the lingual gyrus and cuneus. World Neurosurg. (2021) 151:e426-e37. doi: 10.1016/j.wneu.2021.04.050

31. Suzuki Y, Kiyosawa M, Wakakura M, Ishii K. Glucose hypometabolism in the visual cortex proportional to disease severity in patients with essential blepharospasm. Neuroimage Clin. (2019) 24:101995. doi: 10.1016/j.nicl.2019.101995 
32. Baker RS, Andersen AH, Morecraft RJ, Smith CD, A. functional magnetic resonance imaging study in patients with benign essential blepharospasm. J Neuroophthalmol. (2003) 23:11-5. doi: 10.1097/00041327-20030300000003

33. Haslinger B, Noe J, Altenmuller E, Riedl V, Zimmer C, Mantel T, et al. Changes in resting-state connectivity in musicians with embouchure dystonia. Mov Disord. (2017) 32:450-8. doi: 10.1002/mds.26893

34. Huang XF, Zhu MR, Shan P, Pei CH, Liang ZH, Zhou HL, et al. Multiple neural networks malfunction in primary blepharospasm: an independent components analysis. Front Hum Neurosci. (2017) 11:235. doi: 10.3389/fnhum.2017. 00235

35. Popa T, Velayudhan B, Hubsch C, Pradeep S, Roze E, Vidailhet M, et al. Cerebellar processing of sensory inputs primes motor cortex plasticity. Cereb Cortex. (2013) 23:305-14. doi: 10.1093/cercor/bhs016

36. Neugebauer V, Galhardo V, Maione S, Mackey SC. Forebrain pain mechanisms. Brain Res Rev. (2009) 60:226-42. doi: 10.1016/j.brainresrev.2008.12.014
Conflict of Interest: The authors declare that the research was conducted in the absence of any commercial or financial relationships that could be construed as a potential conflict of interest.

Publisher's Note: All claims expressed in this article are solely those of the authors and do not necessarily represent those of their affiliated organizations, or those of the publisher, the editors and the reviewers. Any product that may be evaluated in this article, or claim that may be made by its manufacturer, is not guaranteed or endorsed by the publisher.

Copyright (c) 2021 Fang, Chen, Chang, Wu and Guo. This is an open-access article distributed under the terms of the Creative Commons Attribution License (CC BY). The use, distribution or reproduction in other forums is permitted, provided the original author(s) and the copyright owner(s) are credited and that the original publication in this journal is cited, in accordance with accepted academic practice. No use, distribution or reproduction is permitted which does not comply with these terms. 